\title{
Clinical and radiological characteristics of pediatric patients with COVID-19: focus on imaging findings
}

\author{
Afshin Mohammadi ${ }^{1}$ Iraj Mohebbi ${ }^{2} \cdot$ Kamal khademvatani $^{3} \cdot$ Habibollah Pirnejad $^{4} \cdot$ Javad Mirza-Aghazadeh $^{2}$. \\ Naser Gharebaghi ${ }^{5} \cdot$ Ali Abbasian Ardakani ${ }^{6} \cdot$ Mohammad Mirza-Aghazadeh-Attari $^{7,8}$
}

Received: 19 April 2020 / Accepted: 4 June 2020 / Published online: 13 June 2020

(c) Japan Radiological Society 2020

\begin{abstract}
Purpose CT imaging has been a detrimental tool in the diagnosis of COVID-19, but it has not been studied thoroughly in pediatric patients and its role in diagnosing COVID-19.

Methods 27 pediatric patients with COVID-19 pneumonia were included. CT examination and molecular assay tests were performed from all participants. A standard checklist was utilized to extract information, and two radiologists separately reviewed the CT images.

Results The mean age of patients was $4.7 \pm 4.16$ (mean \pm SD) years. Seventeen patients were female, and ten were male. The most common imaging finding was ground-glass opacities followed by consolidations. Seven patients had a single area of involvement, five patients had multiple areas of involvement, and four patients had diffuse involvement. The sensitivity of CT imaging in diagnosing infections was $66.67 \%$. Also, some uncommon imaging findings were seen, such as a tree-inbud and lung collapse.

Conclusion CT imaging shows less involvement in pediatric compared to adult patients, due to pediatric patients having a milder form of the disease. CT imaging also has a lower sensitivity in detecting abnormal lungs compared to adult patients. The most common imaging findings are ground-glass opacities and consolidations, but other non-common imaging findings also exist.
\end{abstract}

Keywords Coronavirus infections $\cdot$ Pediatrics · Tomography $\cdot$ X-ray computed $\cdot$ COVID-19 $\cdot$ SARS-CoV-2

Mohammad Mirza-Aghazadeh-Attari

m.aghazadeh75@yahoo.com

1 Department of Radiology, Urmia University of Medical Sciences, Urmia, Iran

2 Social Determinants of Health Research Center, Urmia University of Medical Sciences, Urmia, Iran

3 Department of Cardiology, Faculty of Medicine, Urmia University of Medical Sciences, Urmia, Iran

4 Health Information Technology Department, Urmia University of Medical Sciences, Urmia, Iran

5 Department of Infectious Disease, Faculty of Medicine, Urmia University of Medical Science, Urmia, Iran

6 Medical Physics Department, School of Medicine, Iran University of Medical Sciences (IUMS), Tehran, Iran

7 Aging Research Institute, Tabriz University of Medical Sciences, Tabriz, Iran

8 Medical Radiation Sciences Research Center, Faculty of Medicine, Daneshgah Blvd, Tabriz University of Medical Sciences, Tabriz, Iran

\section{Introduction}

The coronavirus infectious disease 2019 (COVID-19) can cause viral pneumonia coupled with lower respiratory tract symptoms and systemic symptoms [1]. Initial studies suggested that the immune system plays a detrimental role in the pathogenesis of coronavirus and children are not severely affected by a coronavirus $[2,3]$. Other studies suggested that although children may not be affected by the virus as common as the adult population because of differences in the function of the immune system, rather they may act as silent carriers $[4,5]$. Accordingly, a small percentage of pediatric patients suffer from severe disease or lose their lives [6].

The gold standard test for the diagnosis of COVID-19 is reverse transcriptase polymerase chain reaction (RT-PCR); however, studies suggest that CT imaging may have a higher sensitivity and prognostic value [7]. Indeed, other causes of pneumonia may also rise to suspicion based on CT findings [8-10]. As the severity of COVID-19 pneumonia is 
lower in pediatric patients, imaging findings, the pattern of involvement and the role of CT imaging may differ from adult patients.

Few studies discussed the radiologic findings of pediatric patients due to a limited number of them. In the present study, we have evaluated the clinical and radiological characteristics of pediatric patients with COVID-19 pneumonia.

\section{Patients and methods}

This study was approved by the local Institutional Review Board of the University in which it was conducted. All parents of patients had signed informed consent notes before hospitalization and the study was in accordance with the latest issue of the Helsinki Declaration.

\section{Patients}

All of the pediatric patients with clinically diagnosed respiratory tract symptoms who came to our University-affiliated hospitals between January 25th and March 25th were initially included in this study. All of the patients were either directly referred from an internist or infectious disease specialist, or initially came to the emergency department.

The inclusion criteria were as follows: patients with positive RT-PCR results pneumonia, and patients who underwent chest CT examination in the initial day of hospitalization. Those parents who refused written informed consent were excluded.

\section{Molecular assay}

Three samples were obtained from the nasopharynx and oropharynx. Testing was based on the latest guidelines issued by the World Health Organization [11]. RT-PCR was done utilizing a previously approved diagnostic kit by the Chinese center for disease control, targeting the ORFlab and $\mathrm{N}$ genes [12]. The molecular assay was done in a subordinate laboratory of the centers. External validation was obtained from the centers for control of infectious diseases of the country in which the study was performed.

\section{Imaging acquisition and radiological feature extraction}

Patients underwent an imaging protocol based on WHO guidelines and guidelines provided by the Ministry of Health and Education of the country [13]. Imaging was done after consultation with internists and infectious disease fellows. CT examinations of all patients were performed on the initial day of admission via Siemens SOMATOM (Hannover, Germany) and Toshiba Alexion (Tokyo, Japan) scanner based on the following protocol: low dose mode, automatic tube current modulation with a voltage of $120 \mathrm{kVp}$, matrix size of $512 \times 512$, increment and thickness of $1.5-2 \mathrm{~mm}$. CT images were reconstructed in both transverse and sagittal planes. Analysis of the CT slices was done by two separate radiologists with at least 10 years of experience in thoracic imaging according to a checklist adapted from the recommendations of the radiological society of North America (RSNA) and by Kanne et al. [14, 15]. Interpretation of imaging findings was done without prior knowledge of RT-PCR results.

The following imaging findings were extracted from CT images: location of the involvement, in regards to the lobe and pattern of distribution. Distribution as peripheral, central and both central and peripheral, number of lesions, the existence of ground-glass opacity, which was defined as hazy augmented lung attenuation with the maintenance of bronchial and vascular borders, existence of consolidation which was described as opacification with obscuration of vessels and airway borders walls, presence of reticular opacities, defined as every thin linear opacity between $1-3 \mathrm{~mm}$ thickness, nodular lesions which are defined as every round or oval well-marginated opacity, vascular thickening, septal thickening, and bronchial wall thickening, air bronchogram, cavity, cyst, crazy paving, defined as a linear pattern superimposed on a background of ground-glass opacity, resembling irregularly shaped paving stones, halo sign, reversed halo sign, pleural effusion defined as blunting of the costophrenic angle, pleural thickening, lymphadenopathy, and atelectasis.

\section{Statistical analysis}

Two-tailed independent samples $t$ test was used to evaluate age differences between male and female pediatric patients. SPSS software (version 21.0.0, IBM Corporation, Armonk, NY, USA) was used to perform statistical analysis.

\section{Results}

Overall, 27 patients were included in the present study. Seventeen patients were female and 10 patients were male. The mean age of the patients was $4.7 \pm 4.16($ mean $\pm S D)$ years. No significant difference is seen in the mean age of male and female patients $(3.99 \pm 3.91$ vs. $5.16 \pm 4.36 ; P=0.493)$.

All patients showed infection symptoms. The most common symptoms were coughs, fever, and dyspnea. Symptoms of patients on initial presentation are listed in Table 1.

Nine out of 27 patients had non-significant CT findings. Eighteen patients had CT findings suggestive of infection. The most common CT findings were consolidation and ground-glass opacity, which occurred in $10(37 \%)$ and 8 (29\%) patients, respectively. Diffuse, multiple, and single 
Table 1 Clinical signs and symptoms in patients being included in the study

\begin{tabular}{llll}
\hline Clinical sign & Frequency & Clinical sign & Frequency \\
\hline Coughs & $23(85.1 \%)$ & Sore throat & $4(14.8 \%)$ \\
Fever & $16(59.2 \%)$ & Nasal congestion & $0(0.0 \%)$ \\
Dyspnea & $16(59.2 \%)$ & Diarrhea & $1(3.7 \%)$ \\
Malaise & $0(0.0 \%)$ & Nausea & $4(14.8 \%)$ \\
Body ache & $1(3.7 \%)$ & Headache & $2(7.4 \%)$ \\
Irritability & $2(7.4 \%)$ & Chest pain & $2(7.4 \%)$ \\
\hline
\end{tabular}

Table 2 CT imaging findings of patients being included in the study

\begin{tabular}{llll}
\hline Radiologic finding & Frequency & Radiologic finding & Frequency \\
\hline Ground-glass opacities & $8(29 \%)$ & Air-bronchogram & $6(22.2 \%)$ \\
Consolidation & $10(37 \%)$ & Cystic lesions & $1(3.7 \%)$ \\
Reticular lesion & $2(7.4 \%)$ & Cavity & $0(0.0 \%)$ \\
Vascular thickening & $1(3.7 \%)$ & Crazy paving & $1(3.7 \%)$ \\
Nodules & $3(11.1 \%)$ & Halo sign & $0(0.0 \%)$ \\
Septal thickening & $1(3.7 \%)$ & Reverse halo sign & $0(0.0 \%)$ \\
Bronchial wall thicken- & $1(3.7 \%)$ & Pleural effusion & $3(11.1 \%)$ \\
$\quad$ ing & & & \\
Lymphadenopathy & $0(0.0 \%)$ & Pleural thickening & $3(11.1 \%)$ \\
\hline
\end{tabular}

involvements were found in four (15\%), five (19\%) and seven (26\%) patients, respectively. Central and peripheral involvements were observed in one and eight patients, respectively. In addition, seven patients indicated both central and peripheral involvement. Twelve and eleven patients had involvement in lower and upper lobes, respectively. Nine patients had bilateral involvement and seven had unilateral involvement. More details about the CT findings of patients are presented in Table 2. CT sample images of pediatric patients with consolidations and other CT findings are shown in Figs. 1, 2, 3, 4, and 5.

18 out of 27 COVID-19 patients showed positive findings in CT images (sensitivity, 66.67\%). Scoring was done on patients with abnormal findings. One patient had a score of 25 , and 10 patients had a score equal to or less than 6 . The mean score of pediatric patients was $7.5 \pm 6.8$ (mean $\pm \mathrm{SD}$ ).

3 out of 27 patients underwent mechanical ventilation, and the rest were treated with nasal high-flow oxygen or oxygen delivered via an oxygen hood. Of all the patients, two had malignancies (acute lymphoblastic leukemia), four patients had a history of seizures and were treated for the condition, and one patient had chronic kidney disease. Moreover, one patient had previously known exposure to a COVID-19 case in a medical setting, and four had previous close contact with an individual with COVID-19 in their families.

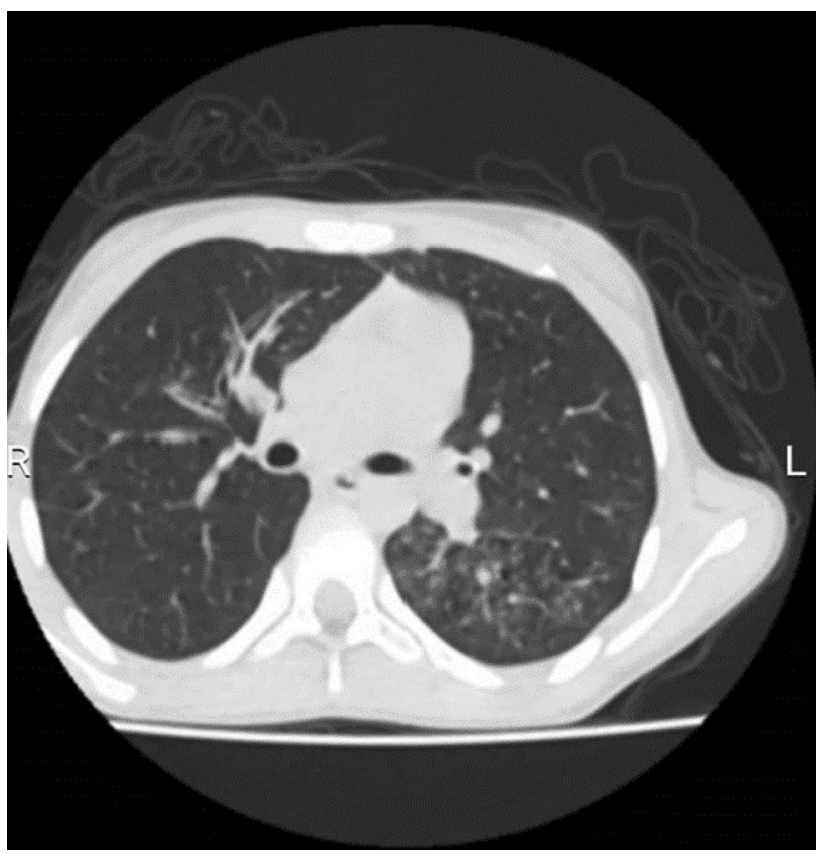

Figure 1 Axial non-contrast computed tomography of an 11-year-old girl shows tree-in-bud nodular appearance of the upper segment of the left lower lobe

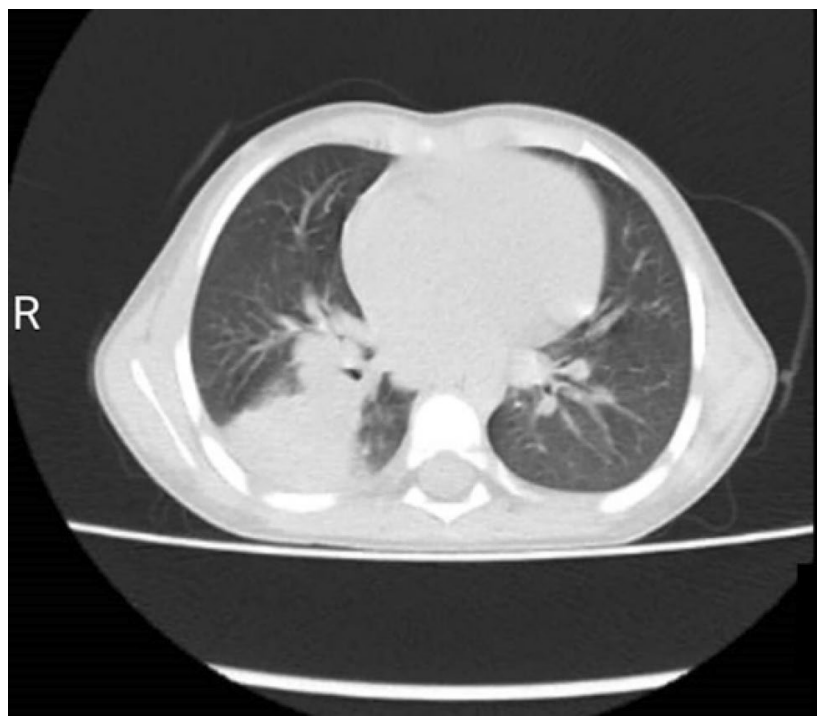

Figure 2 Axial non-contrast computed tomography of a 5-year-old boy shows segmental airspace consolidation of the upper segment of the right lower lobe

\section{Discussion}

CT imaging is an essential diagnostic tool in managing COVID-19 pneumonia. Previous studies showed that CT is a sensitive tool in the diagnosis of symptomatic patients 


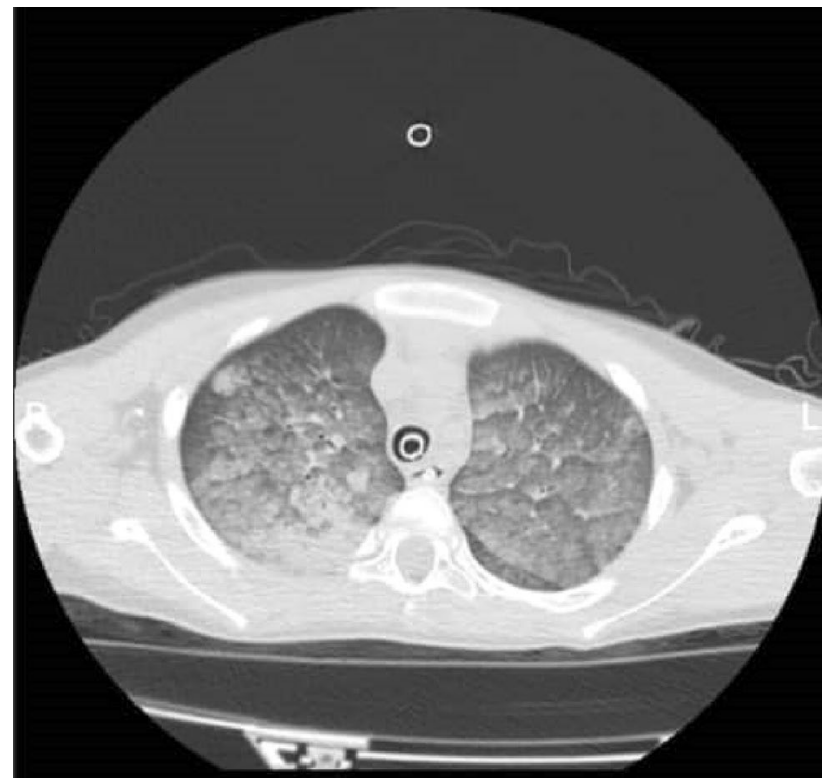

Figure 3 Axial non-contrast computed tomography of an 8-year-old girl shows bilateral diffuse ground-glass opacities

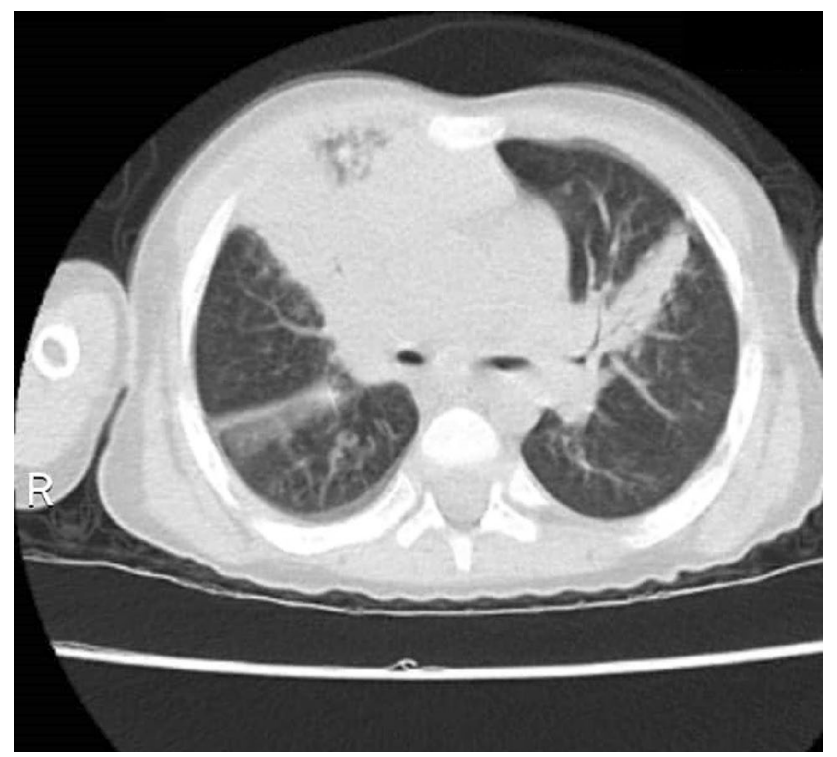

Figure 4 Axial non-contrast computed tomography of a 2-year-old boy shows a patch of air space consolidation in the left upper lobe and collapse consolidation of the right middle lobe

with COVID-19 pneumonia. The most common CT findings in adult patients are peripheral ground-glass opacity and mixed ground-glass opacity. Other CT findings such as air bronchogram, lymphadenopathy, and effusion are less common [16].

As most of the COVID-19 patients being hospitalized are adults, pediatric patients are not discussed extensively in the

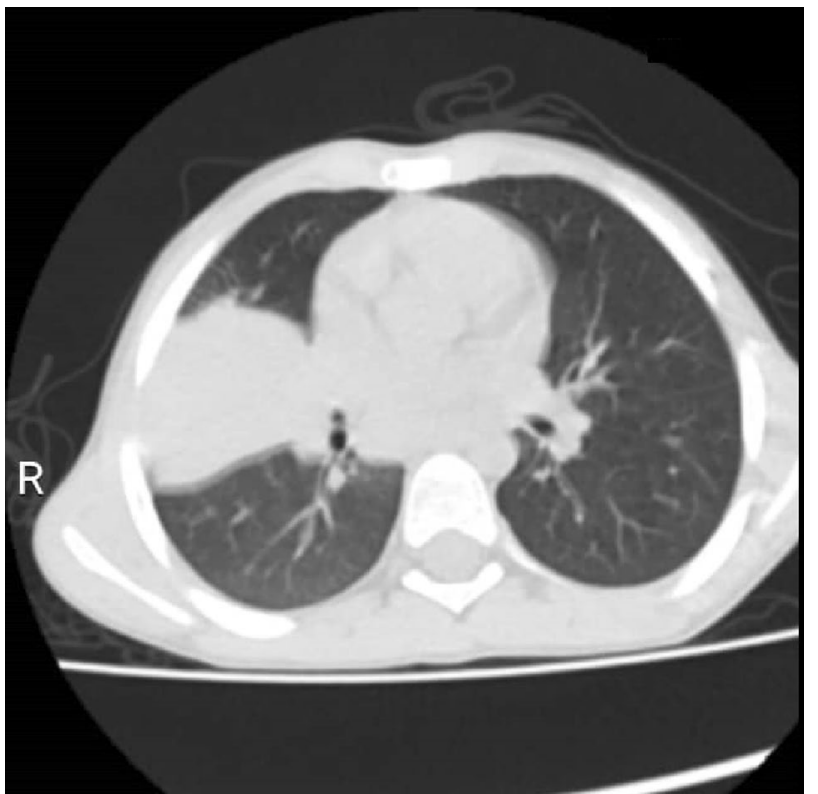

Figure 5 Axial non-contrast computed tomography of a 7-year-old boy shows air space consolidation in the lateral segment of the right middle lobe

literature. Li et al. gave one of the first reports focusing on pediatric imaging findings. They included five patients in their study and found that three of them had patchy groundglass opacities, and two had normal CT findings. Follow-up CT imaging of these three patients with initial abnormal findings was done 5-7 days after the first $\mathrm{CT}$ and was normal in all three [17]. Although a limited number of patients were included in their study, it showed that ground-glass opacity may be the most common imaging finding in pediatric patients, resembling adult subjects.

Another more comprehensive observation was made by $\mathrm{Xia}$ et al. on twenty pediatric patients with the novel coronavirus. They found that 16 patients had involvement (10 bilateral and 6 unilateral), while four patients had normal $\mathrm{CT}$. The most common radiologic finding was ground-glass opacity, which was seen in $12(60 \%)$ patients, and consolidation was seen in $10(50 \%)$ patients. They found nodules and fine mesh shadow in three and four patients, respectively. Their results highlight an important factor which is in line with our findings. In this regard, consolidation is much more common in pediatric patients compared to adult patients. Interestingly Xia et al. witnessed co-infection in eight patients. This can contribute to the various atypical findings seen in pediatric CT imaging [18].

Chen et al. compared CT findings of pediatric with adult patients. They found that fever and severe form of the disease are less common in pediatric patients. In concordance to this, CT imaging of pediatric patients yielded less significant findings compared to adults. They found 
that CT findings were abnormal only in 50 percent of the pediatric patients, while findings were abnormal in $91.5 \%$ of adults. The lung severity score in the adult and pediatric patients was $8.89 \pm 4.54$, and $1.86 \pm 2.41$, respectively $(P<0.05)$. Most of the pediatric patients had one or two lobes involved with the virus $(85.8 \%)$, while most of the adults had three or four involved lobes $(62.8 \%)$. Furthermore, more adult patients had bilateral involvement compared to pediatric patients (83.7 vs. $57.1 \%$ ). Peripheral and peribronchial distributions were more common in adults and pediatric patients, respectively. All of these findings were in accordance with our findings. Hence, we can conclude that pediatric patients have a mild involvement compared to adults. However, Chen et al. found a single case with consolidations, while four patients showed signs of ground-glass opacities. In our study, consolidation was more common, even more, common than ground-glass opacity. The most significant factor contributing to this disagreement maybe that they included a small number of pediatric patients ( 7 in Chen et al. vs. 27 in our study) [19].

A study by Sun et al. examined radiological and clinical findings in eight patients with severe coronavirus disease. Six patients had bilateral pneumonia, and two had unilateral pneumonia. Six patients had ground-glass opacities, seven had patchy involvement, one had pleural effusion, and one patient had white lung change [20]. In their study, only patients with severe involvement were included. Therefore, their findings were comparable to adult patients, as most pediatric patients had ground-glass opacities in both lungs. The results of this study provide evidence that the difference seen in CT findings in adult and pediatric patients may arise from the differences in severity of the disease.

The currently available evidence suggests that patterns of involvement and characteristic of lesions may be different in age groups. We observed tree-in-bud and collapse in the lung in our patients with a severe stage of the disease. Witnessing tree-in-bud appearance is of clinical interest as it shows an endobronchial spread of infection, or even be a sign of the involvement of pulmonary arterioles or interstitium. Noteworthy, clinical studies in recent years have shown that a wide array of conditions ranging from infectious origin to autoimmune ones can cause this specific radiologic sign [21].

These findings were not previously reported in pediatric patients and may either arise from the virus itself or caused by another infection. Although in our study, none of the patients had clinical or laboratory signs of another infection, such as specific clinical signs of any other viral or bacterial disease, positive blood cultures, cultures of lavage samples or specific alterations in blood biochemistry, peripheral blood smear or cell counts to suggest a culprit micro-organism, and thus, we attribute these findings to the COVID-19 infection. Furthermore, the children with rather uncommon imaging signs (including tree-inbud) were all otherwise healthy and did not have any preexisting conditions such as immune dysregulation, asthma, or cystic fibrosis. But it should be mentioned that these patients may have had concomitant infections with difficult to recognize viruses, and our assays do not completely rule out the possibility of concomitant infection.

Another important issue raised by clinicians is that if radiologic findings correspond to pre-existing conditions. In our study, two patients had a history of malignancy (patients 3 and 4). One of them had a lung score of 25 (patient 3) and the other one had a lung score of 3. One patient had chronic kidney disease (patient 15) with a lung score of 4 . The lung score for the four patients with a history of seizure (patients $13,15,16$, and 20) ranged from 0 to 4 . Of all of the patients with pre-existing conditions, patient 3 underwent mechanical ventilation, and the other ones were treated with high-flow nasal oxygen or oxygen delivered by a hood. Our study does not clearly show a relation between the pre-existence of certain conditions and worse clinical outcomes or greater extent of lung involvement. But interpretation should be made with caution as our sample size is limited and we did not intend to compare children with adults in regards to the role of pre-existing conditions in disease severity. A clear understanding in this regard will be achieved with large-scale multi-center studies comparing adults and children with specific conditions such as malignancies, renal disease, hepatic disease, neurologic conditions, etc.

\section{Conclusion}

Radiologic findings are not as common as adult patients in pediatric COVID-19 subjects, and the severity of involvement was less compared to adult patients. Consolidations were more common in pediatric patients and were the most common finding observed in our series of cases. The second most common radiologic finding was ground-glass opacities. In the present study, we observed a tree-in-bud formation and two incidences of segmental collapse, not previously reported for COVID-19 patients. Our results suggest that pediatric patients should not be evaluated by diagnostic criteria specifically designed for an adult population.

Author contributions All authors contributed equally.

\section{Compliance with ethical standards}

Conflict of interest The authors declare no conflict of statement. 


\section{References}

1. Ahmadzadeh J, Mobaraki K, Mousavi SJ, Aghazadeh-Attari J, Mirza-Aghazadeh-Attari M, Mohebbi I. The risk factors associated with MERS-CoV patient fatality: a global survey. Diagn Microbiol Infect Dis. 2020;96(3):114876.

2. Lipsitch M, Swerdlow DL, Finelli L. Defining the epidemiology of Covid-19 - studies needed. N Engl J Med. 2020;382(13):1194-6.

3. Aghazadeh-Attari J, Mohebbi I, Mansorian B, Ahmadzadeh J, Mirza-Aghazadeh-Attari M, Mobaraki K, et al. Epidemiological factors and worldwide pattern of Middle East respiratory syndrome coronavirus from 2013 to 2016. Int J Gen Med. 2018;11:121.

4. Cristiani L, Mancino E, Matera L, Nenna R, Pierangeli A, Scagnolari $\mathrm{C}$, et al. Will children reveal their secret? The coronavirus dilemma. Eur Respir J. 2020:200.

5. Bai Y, Yao L, Wei T, Tian F, Jin D-Y, Chen L, et al. Presumed asymptomatic carrier transmission of COVID-19. JAMA. 2020;323(14):1406-7.

6. Kim DD, Goel A. Estimating case fatality rates of COVID-19 [published online ahead of print, 2020 Mar 31]. Lancet Infect Dis. 2020;S1473-3099(20)30234-6. https://doi.org/10.1016/S1473 -3099(20)30234-6.

7. Fang Y, Zhang H, Xie J, Lin M, Ying L, Pang P, et al. Sensitivity of Chest CT for COVID-19: Comparison to RT-PCR. Radiology. 2020:200432.

8. Saleh P, Taghizadeh S, Piri R, Mohammadi S, Naghavi-Behzad M. Radiological findings in patients with H1N1: a report from the Referral Center of Northwest of Iran. Shiraz E Med J. (In Press).

9. Kanne JP, Yandow DR, Meyer CA. Pneumocystis jiroveci pneumonia: high-resolution CT findings in patients with and without HIV infection. Am J Roentgenol. 2012;198(6):W555-W56161.

10. Kim J, Lee IJ, Kim J-H. CT findings of pulmonary tuberculosis and tuberculous pleurisy in diabetes mellitus patients. Diagn Interv Radiol. 2017;23(2):112.

11. Organization WH. Laboratory testing for coronavirus disease 2019 (COVID-19) in suspected human cases: interim guidance, 2 March 2020. Geneva: World Health Organization; 2020.
12. Corman V, Bleicker T, Brünink S. Diagnostic detection of Wuhan coronavirus 2019 by real-time RT-PCR. Geneva: World Health Organization; 2020.

13. Organization WH. Clinical management of severe acute respiratory infection when novel coronavirus (nCoV) infection is suspected: interim guidance, 25 January 2020. Geneva: World Health Organization; 2020.

14. Kanne JP. Chest CT Findings in 2019 Novel Coronavirus (2019$\mathrm{nCoV}$ ) Infections from Wuhan, China: key points for the Radiologist. Radiology 2020;295(1):16-7.

15. Kanne JP, Little BP, Chung JH, Elicker BM, Ketai LH. Essentials for Radiologists on COVID-19: An Update-Radiology Scientific Expert Panel. Radiology 0(0):200527.

16. Liu H, Liu F, Li J, Zhang T, Wang D, Lan W. Clinical and CT imaging features of the COVID-19 pneumonia: Focus on pregnant women and children. J Infect. 2020;80(5):e7-e13.

17. Li W, Cui H, Li K, et al. Chest computed tomography in children with COVID-19 respiratory infection. Pediatr Radiol. 2020;50:796-9. https://doi.org/10.1007/s00247-020-04656-7.

18. Xia W, Shao J, Guo Y, Peng X, Li Z, Hu D. Clinical and CT features in pediatric patients with COVID-19 infection: different points from adults. Pediatr Pulmonol. 2020;55(5):1169-74.

19. Chen A, Huang J, Liao Y, Liu Z, Chen D, Yang C, et al. Differences in clinical and imaging presentation of pediatric patients with COVID-19 in comparison with adults. Radiol Cardiothorac Imaging. 2020;2(2):e200117.

20. Sun D, Li H, Lu X, et al. Clinical features of severe pediatric patients with coronavirus disease 2019 in Wuhan: a single center's observational study. World J Pediatr. 2020. https://doi. org/10.1007/s12519-020-00354-4.

21. Miller WT Jr, Panosian JS. Causes and imaging patterns of treein-bud opacities. Chest. 2013;144(6):1883-922.

Publisher's Note Springer Nature remains neutral with regard to jurisdictional claims in published maps and institutional affiliations. 